\title{
Blind Distributed MU-MIMO for IoT Networking over VHF Narrowband Spectrum
}

\author{
Chuhan Gao \\ University of Wisconsin-Madison \\ chuhan@cs.wisc.edu \\ Krishna Chintalapudi \\ Microsoft Research \\ krchinta@microsoft.com
}

\author{
Mehrdad Hessar \\ University of Washington \\ mehrdadh@uw.edu \\ Bodhi Priyantha \\ Microsoft Research \\ bodhip@microsoft.com
}

\begin{abstract}
Longer range, in rural/urban IoT networks, allow a large geographical coverage with only a few base-stations, making their deployment and operation economical. In this paper we explore the 150-174 MHz spectrum for long range IoT networks comprising unlicensed MURS and licensed VHF narrowbands. Range in these bands is boosted by the lower RF frequencies as well as higher transmit powers allowed by the FCC. Through a $400 \mathrm{sq} \mathrm{km}$ wide area deployment study, we show that, these spectrum bands can provide $>20 \times$ the geographical coverage than that in the $900 \mathrm{MHz}$ ISM band LoRa.

Increased range translates to greater uplink IoT device traffic. The key contribution of this paper is a novel technique - Blind Distributed MU-MIMO, that allows capacity to scale with the number of antennas (basestations) while not requiring any coordinated channel measurements between the devices and IoT basestations. This requirement is crucial since in IoT networks power constrained IoT devices typically sleep and wake up to transmit short messages in response to unpredictable events without any coordination with the base-stations. We demonstrate the efficacy of Blind Distributed MU-MIMO through a real wide area deployment.
\end{abstract}

\section{KEYWORDS}

Wireless; LPWAN; Distributed MIMO; MU-MIMO
ACM Reference Format:

Chuhan Gao, Mehrdad Hessar, Krishna Chintalapudi, and Bodhi Priyantha. 2019. Blind Distributed MU-MIMO for IoT Networking over VHF Narrowband Spectrum. In The 25th Annual International Conference on Mobile Computing and Networking (MobiCom '19), October 21-25, 2019, Los Cabos, Mexico. ACM, New York, NY, USA, 17 pages. https://doi.org/10.1145/3300061.3345427

\section{INTRODUCTION}

A large number of urban/rural IoT applications (e.g. smart agriculture, smart cities etc.) require long range wireless connectivity to the IoT devices. Longer range helps cover a vast geographical area with fewer base-stations and consequently lowers network deployment and operational costs. Existing wide area IoT networks use either the unlicensed ISM bands [4] or rely on cellular connectivity. In this paper, we explore the use of 150-174 MHz VHF spectrum comprising unlicensed Multi-Use Radio Service (MURS) [5] and licensed VHF narrowband channels [2] for IoT networking. The key advantage of using these bands is a significantly superior range fueled by two factors $-200 \times$ higher transmit power allowance by FCC in these bands compared to that in the ISM bands and, superior radio propagation characteristics at 150-174 MHz frequencies.

Order of Magnitude Higher Coverage. We have deployed an IoT network convering $400 \mathrm{~km}^{2}$ of urban/sub-urban areas with a traffic of $\approx 300,000$ uplink messages/day operating over the MURS bands. Our measurements demonstrate that an IoT base-station operating in MURS bands can provide > $20 \times$ the geographical area coverage compared to that in 900 MHz ISM spectrum using LoRa.

Challenge of Network Capacity and Distributed MIMO. This increased geographical coverage translates to a larger number of IoT devices and consequently the need to accommodate higher uplink network capacity. Given the limited amount of licensed/unlicensed spectrum available in these bands $(\approx 24 \mathrm{MHz})$, distributed MIMO [14, 18, 39, 40, 58] provides the promise of scaling capacity by enabling basestations to collaboratively decode concurrent transmissions. 
Need for Blind Distributed MU-MIMO (Iris). In order to enable simultaneous decoding of multiple concurrent transmissions using distributed MIMO, the channel parameters and carrier frequency offsets (CFO) for all participating transmitter-receiver pairs must be known. Thus, communication in existing systems alternates between a measurement phase and a transmission phase. In the measurement phase, all participating transmitters and receivers coordinate to first conduct channel measurements and then all transmitters transmit concurrently. Expecting this manner of coordination between IoT devices and base-stations, as well as the resulting measurement phase overheads is not a viable option for IoT networks. This is because, a typical IoT network comprises power constrained IoT devices that predominantly sleep (turn radios off) and sporadically wake up on the occurrence of events to transmit short notification messages (typically a few bytes). In this paper we present a novel technique named Iris- Blind Distributed Multi-User MIMO, that eliminates the need for coordination and a measurement phase; IoT devices may simply transmit at any time as usual.

How Iris Works Iris leverages a key property of MURS and VHF narrowband channels - unlike wider channels, they can be represented by a single complex number (since the delay spread of the channel is much smaller than the required Nyquist sampling rate). Concurrent transmissions from IoT devices (note that transmissions may all have started at different points in time from different IoT devices) superimpose in the wireless channel and are received at various basestations. The base-stations then forward received IQ samples to a central entity in the cloud. Using the received IQ samples and the known preamble IQ samples, and the unknown channels, CFOs and undecoded symbols, Iris forms and solves a set of non-linear equations in real time. This allows Iris to decompose the received superimposed signals into the IQ sample streams corresponding to individual IoT devices. Once the streams of IQ samples for the various transmissions are separated, the decoder decodes each stream to extract the data in each packet. This ability of Iris to separate out IQ samples from individual concurrent streams allows it to perform distributed MIMO even for non-linear and phase non-coherent modulation schemes such as FSK which is mandated by the FCC in MURS bands.

\section{Summary of Contributions.}

- We present the first measurement study for an IoT network in the MURS band using a pilot deployment spanning $400 \mathrm{~km}^{2}, 90$ mobile IoT devices transmitting 300,000 messages/day. Our deployment demonstrates $20 \times$ greater coverage, compared to LoRa at $900 \mathrm{MHz}$.

- We propose a novel blind distributed multiuser MIMO technique that allows base-stations to perform distributed
MIMO and receive concurrent uplink transmissions without requiring any coordinated measurements with the devices. Iris can even work with non-linear and noncoherent modulations e.g. FSK since it can separates concurrent transmissions at the IQ sample level.

- We evaluate Iris using a real deployment and demonstrate that it is practical. Having the advantage of a real large scale IoT network deployment.

\section{MURS BAND - MEASUREMENT STUDY}

The unlicensed MURS band [5], created in 2000 by FCC, comprises 5 channels (three $11.5 \mathrm{kHz}$ and two $20 \mathrm{kHz}$ channels) in the $150 \mathrm{MHz}$ spectrum. The licensed VHF narrowbands [2] in 150-174 MHz, have been traditionally used for handheld radio communication for public safety and industrial/business applications over $25 \mathrm{kHz}$ channels. As of 2013, the FCC narrowbanding mandate [20] requires that these channels be $12.5 \mathrm{kHz}$ and can be used for two way data or voice communication. These licensed VHF narrowband channels can be leased in sets of 2 channels for a period of 10 years by applying to FCC. In this section we use a measurement study to argue that these bands can provide a significantly higher range compared to ISM bands as well as whitespaces, making it extremely economical to deploy wide area IoT networks. To the best of our knowledge, there has been no prior work studying these bands for IoT applications.

MURS and VHF Narrowband Regulations. FCC allows IoT devices to transmit data at $2 \mathrm{~W}$ in these narrowband channels $(200 \times$ higher than the maximum $100 \mathrm{~mW}$ allowed in ISM bands and whitespaces). The regulation also mandates the use of FM/FSK modulation for MURS band with a maximum allowed frequency deviation.

\subsection{Deployment and Measurement Study}

We partnered with a well known field services company to pilot a large scale IoT network over the MURS band. The company provides several services such as food catering, water and beverage distribution, furniture movement etc. over nine cities covering $\approx 400 \mathrm{~km}^{2}$ of geographical area using a fleet of vehicles. We used the Raveon RV-M7-VM-GX [10] MURS band modem to build a vehicle tracking device (Fig. 1) that transmits vehicle latitude, longitude, altitude, heading, speed etc. over the MURS channels at $2 \mathrm{~W}(33 \mathrm{dBm})$ transmit power and 4800bps. We deployed these devices on 90 vehicles. The vehicles transmit once every 10 seconds, only when there is a "significant" change ( $1 \mathrm{~m}$ or more) in their location. The base-stations do not perform any downlink transmissions or acknowledgements, as reporting GPS data can tolerate occasional packet loss. Our base-stations were also built using the same transceiver and located on roof-tops about $20 \mathrm{~m}$ high above the ground. The network transmits 

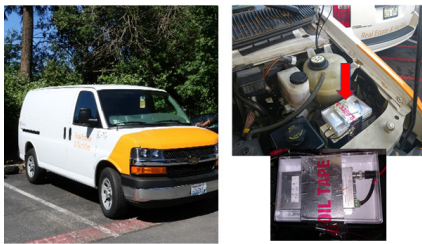

Figure 1: Vehicles and MURS IoT device.

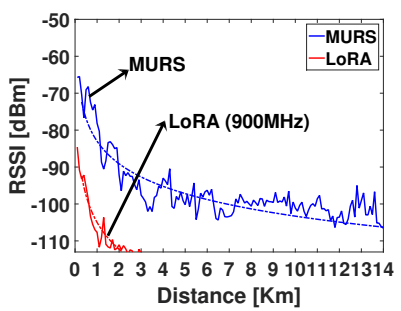

Figure 5: MURS and LoRa band path loss curve.
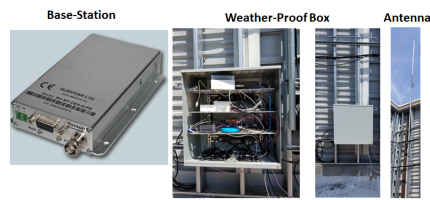

Figure 2: Base-station and antenna on roof top

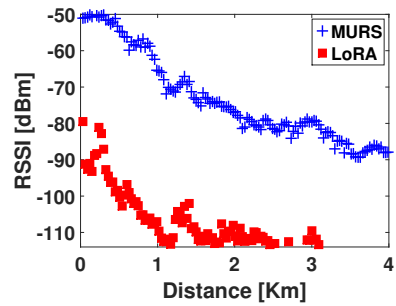

Figure 6: Drive compariso of MURS and LoRa.

over $\approx 300,000$ IoT messages each day and has been in use by the company for the past one year. Fig. 3 shows a screenshot of the real-time view.

Range of MURS. Fig. 5 depicts the dependence of mean RSSI (over measurements from all 90 vehicles) with distance for MURS transmissions plotted using 3,000,000 measurements collected over a period of ten consecutive days from the vehicles in our deployment from one of the base-stations. To plot Fig. 5, we collected all RSS measurements within distance bins of $100 \mathrm{~m}$ resolution and averaged them. As seen from Fig. 5, the BS has a range of about 13 to $14 \mathrm{~km}$ (range corresponding to $-105 \mathrm{dBm}$ where packet success rate falls below 80\%). Fig. 4 depicts the locations of the vehicles from which this BS received messages on a map.

A Drive Based Direct Comparison With 900 MHz LoRa. To compare MURS and the $900 \mathrm{MHz}$ band LoRa, we used a Dragino LoRa Shield board (equipped with Semtech SX1276 LoRa chip) connected to an Arduino boards as the client. To appropriately compare ranges we kept the data transmission rate of the devices must be similar - our MURS and LoRA devices transmited at $4800 \mathrm{bps}$, and $4557 \mathrm{bps}$ (spreading factor of $7,125 \mathrm{kHz}$ bandwidth, coding rate of 6/4) respectively at their FCC allowed maximum output power limits. We placed a LoRa BS on the same location as the MURS BS used for measurements. We found that LoRa transmissions reception probability fell under $80 \%$ probability at $-113 \mathrm{dBm}$.

We placed a LoRa device and a MURS device both on the same vehicle transmitting the GPS position every second This allowed us to obtain RSSI measurements from both these devices from the same set of geographical locations as the vehicle drives. Fig. 6 depicts the RSSI for each of the

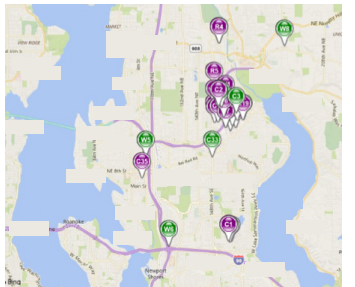

Figure 3: Screenshot of rea time vehicle locations.

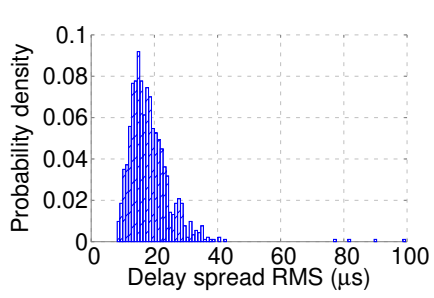

Figure 7: distribution of RMS delay spread.

devices with distance as the vehicle travelled away from the base-station. As seen from Fig. 6, MURS band RSSIs are about 30-40 dB higher than that received by LoRa. In fact, after about $3 \mathrm{~km}$ the LoRa BS stopped receiving transmissions as the signal strength was too low.

Path Loss Curves. In order to measure the coverage of LoRa, we performed several additional drives around areas as far as $10 \mathrm{~km}$ around the BS. Fig. 5 depicts the path loss curve for LoRa obtained from 20,000 measurements, the same way as we did for MURS and has a range of about $2.5 \mathrm{~km}$ (corresponding to $-113 \mathrm{dBm}$ ). Similar LoRa range results have been reported for urban environments by several researchers in the past for urban environments for LoRa in $900 \mathrm{MHz}$ at 4557bps. [51, 57, 70]. We modeled the received power at a distance $d \mathrm{Km}, P(d)$ using the Log Distance Path Loss (LDPL) model given by, $P(d)=P_{0}-10 \gamma \log _{10}(d)$ (in $\mathrm{dBm}$ ) where $P_{0}$ is the received power $1 \mathrm{~km}$ away and $\gamma$ the path loss exponent. $\left\langle P_{0}, \gamma\right\rangle$ for MURS and LoRa were found to be $<-81.6,2.05>$ and $<-104,2.4>$. The higher $\gamma$ indicates the faster decay of transmissions in the $900 \mathrm{MHz}$ band compared to the $150 \mathrm{MHz}$ MURS band. This shows that two factors contribute to MURS band's higher range - higher transmit power allowed by FCC in the MURS band and, the slower path loss decay in the $150 \mathrm{MHz}$ band.

Comparing MURS band and T.V whitespaces. T.V whitespaces can potentially be used for IoT applications [13, 74]. FCC regulations allow a maximum of $20 \mathrm{dBm}(100 \mathrm{~mW})$ over $6 \mathrm{MHz}$ channels. While T.V whitespace frequencies around and below $174 \mathrm{MHz}$, can have similar or better propagation characteristics than MURS band, the $200 \times$ higher transmit power and lower noise floor due to narrowband channels 
provides MURS bands a significant advantage. T.V whitespaces however, offer the advantage of providing significantly higher data rates as they provide up to $6 \mathrm{MHz}$ channels and can be used for IoT applications that require higher data rates e.g. video/audio signals.

\subsection{Multipath in Narrowband Channels}

A key property of using narrowband channels is that they experience no inter-symbol interference (ISI) due to multipath. This is because the multipath delay spread is much smaller than the Nyquist sampling of the narrowband channel. Consider for example, that the Nyquist sampling interval for $12.5 \mathrm{kHz}$ channel is $80 \mu \mathrm{s}$. This means that only reflection paths greater than $27 \mathrm{~km}$ (light travels $1 \mathrm{~km}$ in $3 \mu \mathrm{s}$ ) will cause ISI. Given that the range of the radio is far smaller than $27 \mathrm{~km}$, such large multipaths have power far below the noise floor. Given the absence of ISI, the narrowband wireless channel experiences flat fading and can be represented by a single complex number [19].

In order to experimentally establish the above property, we conducted outdoor delay spread measurements. In our experiments, transmitters transmit 13-sample Barker code continuously at the sampling rate of $12.5 \mathrm{kHz}$ from five outdoor locations to the base-station. The impulse response of the channel is estimated by deconvolving the received signal with the Barker code sequence. We then measure the RMS delay spread [36] of the 100,000 collected impulse responses. The distribution of the delay spread RMS values from different measurements are plotted in Fig. 7. Almost all measured delay spreads fall below $40 \mu \mathrm{s}$, while each sample is $80 \mu \mathrm{s}$ long, thus demonstrating the lack of ISI.

\subsection{Limitations}

We now discuss the limitations of using MURS and VHF narrowbands for long range IoT applications.

Limited Bandwidth (12.5 kHz). The limited bandwidth of these channels (mostly $12.5 \mathrm{kHz}$ ) limits the maximum data rate that may be supported per channel few kbps. This precludes data intensive IoT applications that transmit video or high resolution images for example. However, they are suitable of a large number of IoT applications need only transmit a few bytes e.g. smart utility meters transmit a few bytes of data once every few hours, GPS devices to track people, animals and vehicles and applications that send alarms and messages in response to sporadic events.

Larger Antenna Size. Lower frequencies typically require larger antenna sizes owing to the longer wavelengths. For example, a quarter wavelength whip antenna at $150 \mathrm{MHz}$ will be about $50 \mathrm{~cm}$ long compared to only $8 \mathrm{~cm}$ for ISM 900 $\mathrm{MHz}$. Large antenna sizes increase the form factor of the IoT devices and potentially preclude several applications that require small sized devices. However, it is possible to design compact or viable antennas that specifically designed an application. For example, Fig. 8 shows a MURS vest antenna (PHARAD [12]) that can be worn by public safety personnel or police officers, as well as a $150 \mathrm{MHz}$ phantom antenna (TRAT1500 [11]) that be mounted on quadcopters.

\section{DISTRIBUTED MIMO AND MU-MIMO}

In order to take advantage of the increase in range of the basestation, the network must also be able to support traffic from a larger number of IoT devices. Distributed MIMO performs joint multi-user beamforming enabling independent access points (APs) to beamform their signals, and communicate with their potentially interfering clients concurrently on the same channel as if they were one large MIMO transmitter [14, 18, 39, 40, 58]. Uplink Multi-User MIMO (MU-MIMO) operates in similar principle to support multiple concurrent data streams from a group of users to the AP $[17,35,66]$. Although they have differences, our primarily aim is to enable concurrent uplink transmission, for which distributed MIMO and MU-MIMO share the same principle, thus we discuss them together in this section.

Requirements for enabling distributed and MU-MIMO. (i) In order to enable beamforming, with $N_{T}$ transmitters and $N_{R}$ receivers, all the $N_{R} \times N_{T}$ channels must be known prior to the decoding. (ii) All the $N_{R} \times N_{T}$ CFO values are needed for decoding. (iii) The transmitters need to be coordinated and time synchronized to transmit precoded symbols.

Distributed MIMO and MU-MIMO operation. The operation typically contains two phases. (i) The $N_{T}$ transmitters conduct a coordinated channel estimation phase, where each AP takes turn to send channel and CFO estimation sequence and collect feedback from the $N_{R}$ receivers. An illustration of this process is well presented from Fig. 9, which we borrow from [58]. Later works have managed to reduce the overhead associated with this phase by taking advantage of channel reciprocity, CFO across receivers are fixed and can be known, and etc. [14, 18, 39, 40]. Minimally, CFO and channel estimation involves each transmitter and receiver sending estimation sequence and feedback once in sequence, which incurs an overhead of $N_{T}+N_{R}$ messages. (ii) With the collected channel feedback, the transmitters precode their transmissions, in order to enable concurrent transmissions. Precoding the transmissions require the transmitters to be time synchronized, which is typically enabled by the wired connection among transmitters [58] or transmitting synchronization beacons [35, 40].

Need for Blind MIMO in IoT networks. IoT device radios are configured to be turned off by default to save power. They are turned on to transmit messages to convey the occurrence of sporadic events that occur unpredictably. The 


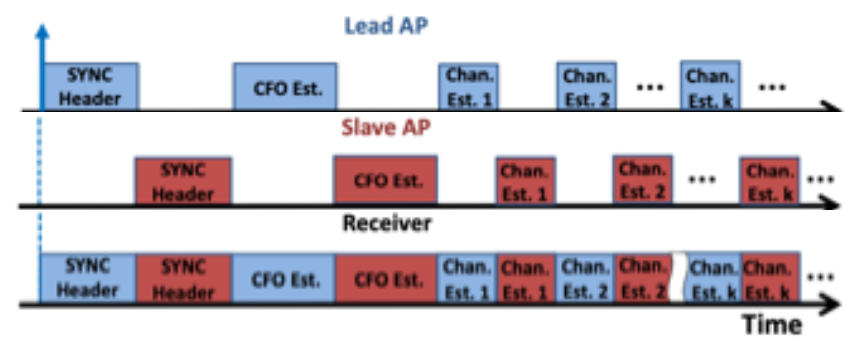

Figure 9: Illustration of distributed MIMO channel estimation phase (originally from [58]).

messages themselves are only a few bytes. Consequently, introducing distributed MU-MIMO in an IoT network faces two key challenges: the impracticality of conducting the coordinated measurement phase with multiple IoT devices and the high signalling overhead. With random triggering sporadic events and mostly turned off radios, requiring a measurement phase where all IoT devices and BS coordinate becomes impractical. Further, distributed MIMO's channel feedback overhead grows rapidly as the network size increases [39]. The problem is already significant in WLANs a $8 \times 8$ distributed MIMO incurs over $20 \%$ signalling overhead in WLAN with $100 \mathrm{~ms}$ feedback interval even with packets with megabytes of data. In IoT where message sizes are only a few bytes, and devices are power constrained, this overhead can be crippling. Thus, in order enable distributed MIMO and achieve high throughput in a long range IoT network, the design must eliminate signalling overhead, and cannot assume any coordination or synchronization among IoT client devices. We illustrate how Iris achieves these two goals in the next section.

\section{IRIS}

Iris completely eliminates the need for a dedicated channel and $\mathrm{CFO}$ measurement phase. It allows devices to transmit as usual, independently and at arbitrary times. The operation of Iris is depicted in Fig. 10. When multiple devices concurrently transmit, the received signal at each base-station is a linear combination of the individual transmissions. The base-stations forward the IQ samples received to a central entity in the cloud that uses Iris to decompose these received signals into individual IQ sample streams from each of the transmitting devices. Given that the sample duration for each sample is $80 \mu$ s for $12.5 \mathrm{KHz}$ channels, the GPSDO is able to synchronize the samples to sub-sample accuracy. The IQ sample streams are then demodulated individually. Since Iris retrieves the signals from each devices at the IQ sample level and the demodulation is performed on the IQ samples, it can be used with any arbitrary modulation scheme, even nonlinear and phase non-coherent modulations such as FSK (as mandated by FCC for MURS bands). The key challenge Iris overcomes is to reliably estimate CFO and channels between the transmitting devices and the receiving base-stations using the preambles while simultaneously recovering the IQ samples of the individual data streams. Iris is amenable to parallel multi-threaded implementation and has been implemented in C and python (details in Sec. 5) allowing it to perform real-time decoding while scaling to a large number of concurrent transmissions.

\subsection{Iris Operation Overview}

Iris has three distinct phases of decoding -idle state, steady state and, blind estimation state. The state diagram is presented in Fig. 11. Iris starts in the idle state corresponding to When there are no ongoing transmissions being received at the base-stations. Upon detecting one or more new preambles indicating new packet transmissions, Iris transitions into the blind estimation state. In this phase, Iris decodes while simultaneously estimating the CFOs and channels between all transmitter-receiver pairs, after which it moves into the steady state. In the steady state, the CFO and channels at each receiver for all the concurrent transmissions have already been estimated, consequently, estimating the next IQ samples for each transmission is akin to standard MIMO decoding (Sec. 4.2).

In the steady state, if a preamble is detected, Iris once again enters the blind estimation state as there one or more new transmissions whose CFO and channels are unknown and it is not possible to estimate the IQ values of the already ongoing transmissions. However, the first few IQ samples of the new transmissions are already known since they are preambles. Iris stalls estimation of IQ samples and decoding until it can accumulate enough information (samples) to allow joint estimation of all the unknown data samples from ongoing transmissions and the CFOs, channel parameters of all the new transmissions. Once it has enough samples, Iris uses blind estimation (Sec. 4.3) to jointly estimate unknown CFOs, channels and the pending unknown data IQ samples. Then once again, having estimated all the channel parameters and CFOs, Iris resumes its steady state decoding. Steady state decoding as well as blind estimation, Iris has to keep track of the number of transmissions by counting the number of detected preambles and the transmissions that ended. Having estimated the IQ samples of each individual transmission, each of the sample streams are demodulated and decoded into symbols. Therefore, Iris is modulation-independent. To provide an insight to the reader, we provide an illustrative example (Fig. 12).

Steady State Example. Here the CFO and channels at each receiver for all the concurrent transmissions have already been estimated. In Fig. 12 (a), there were $N_{T}$ ongoing transmissions, all of whose CFOs and channels have already been 


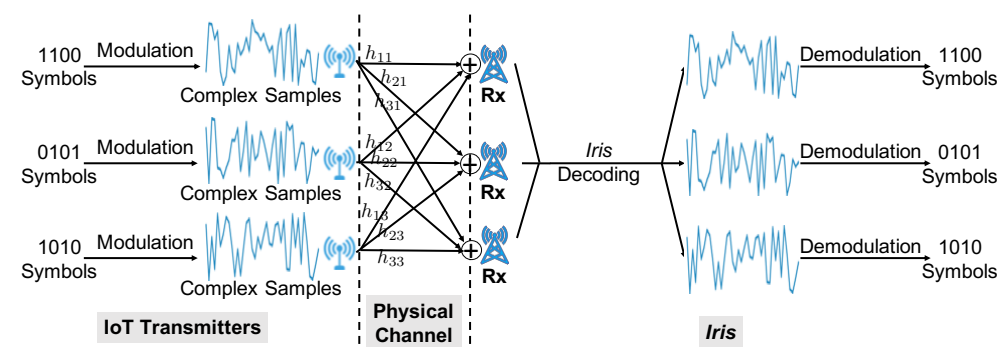

Figure 10: Overview of Iris.

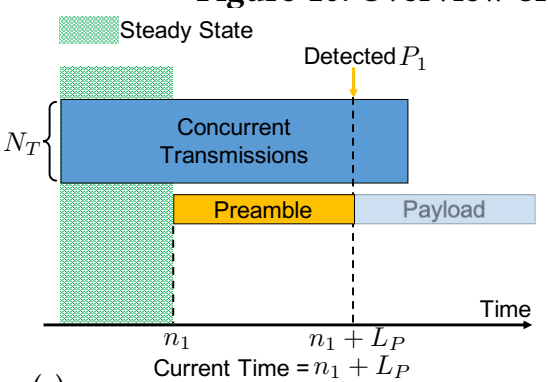

(a)

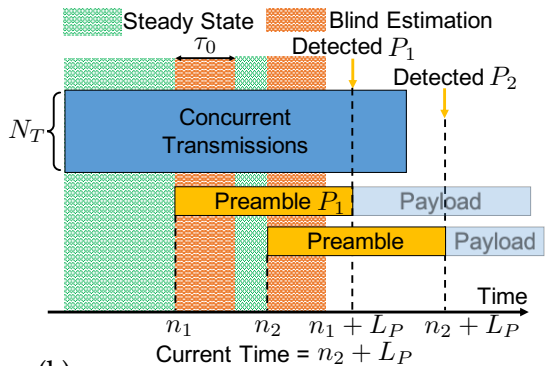

(b)

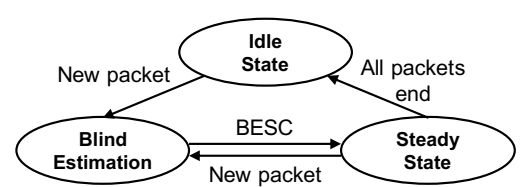

Figure 11: Iris state diagram.

Figure 12: Iris operation illustration. (a) Joint decoding data samples in steady state until a new preamble detected at time $n_{1}+L_{P}$. (b) Blind estimation phase, case 1. (b) Blind estimation phase, case 2.

estimated. A new transmission (Packet $P_{1}$ ) begins at sample $n_{1}$. Until sample $n_{1}-1$, Iris operates in steady state and the samples of all concurrent transmissions can be decoded using steady state decoding described in Sec. 4.2. However, beginning from $n 1$, not knowing the $\mathrm{CFO}$ and channel for the new transmission, Iris can no longer estimate IQ samples of the ongoing transmissions using steady state decoding and enters the blind estimation phase. Note that, Iris will detect the new transmission only after it has received $n_{1}+L_{P}$ th sample, where $L_{P}$ is the length of the packet preamble. In our implementation, we use a Barker code of length 13 samples as a preamble. Thus, Iris can only be certain about the number of active transmissions for samples that were received $L_{P}$ samples before the current sample. Consequently, Iris always has to maintain a buffer of at least $L_{P}+1$ samples.

Blind Estimation Example. Prior to sample $n_{1}$ there were $n_{T}$ unknowns corresponding to the samples of each transmission. At the $n^{t h}$ sample, since the preamble is known, now there are $n_{T}+2 * N_{R}$ unknowns including the new transmissions CFOs and channels. Consequently, Iris must jointly estimate all these variables from the $N_{R}$ received transmissions at $N_{R}$ antennae. In general, $\tau$ samples into the new transmission's preamble i.e. at the $\left(n_{1}+\tau\right)^{t h}$ sample, Iris must jointly estimate $2 * N_{R}+\tau N_{T}$ unknowns corresponding to all the unestimated samples from ongoing transmissions thus far from $N_{R} * \tau$ received samples. At $\tau=\tau_{0}$ samples, Iris has enough samples to solve for all the $2+\tau_{0} N_{T}$ unknowns accumulated thus far. At this point, Iris uses blind estimation to jointly solve for all the unknowns and thus determines the $\mathrm{CFO}$ and channel for the new transmission and resumes to steady state decoding phase. The same sequence repeats when a new preamble is detected again in the steady state phase. This is depicted in Fig. 12 (b).

Arrival of another preamble within $\tau_{0}$ samples. As depicted in Fig. 12 (c), a new transmission (packet $P_{2}$ ) may initiate before $\tau_{0}$ samples i.e before Iris is able to solve for all the pending unknowns. This new transmission adds an extra $2 N_{R}$ unknowns, the corresponding CFOs and channels) to be estimated. This has an effect of increasing the value of $\tau_{0}$, the minimum samples needed before all the unknowns can be solved using blind estimation after which Iris resumes to steady state decoding. Thus, the value of $\tau_{0}$ depends on the number of unknown transmissions that keep arriving during the blind estimation phase.

It is possible that too many new packets arrive too quickly, leaving insufficient samples to estimate their channels. This would cause Iris's decoding to fail. Similar to all MIMO systems, the number of concurrent packets that can be supported is always related to the number of receivers. We discuss this scenario along with other possible failures and their impacts in Sec. 4.5.

\subsection{Steady State Decoding}

The received IQ samples $y_{i}$ at receiver $i$ are a superposition of the signals received from all transmitters that are in range:

$$
y_{i}[n]=\sum_{k}^{N_{T}} h_{i k} \cdot e^{j 2 \pi \Delta f_{i k} n T_{S}} \cdot x_{k}[n]
$$


In Eqn $4.2 n$ is the discrete sample time index, and $T_{s}$ is the sample duration. $x_{k}[n]$ is transmitter $k$ 's complex sample, $\Delta f_{i k}$ and $h_{i k}$ represents the CFO and complex channel between transmitter $k$ and receiver $i$. As described in Sec. 2.2, $h_{i k}$ is a single complex number. Eqn 4.2 is independent of the modulation scheme - thus, it applies to even non-linear modulation schemes such as FSK.

If there are $N_{T}$ concurrent transmissions being received at $N_{R}$ receivers, based on Eqn 1 we have,

$$
\mathbf{x}[n]=\mathbf{H}[\mathbf{n}] \mathbf{x}[n]
$$

In Eqn $2, \mathbf{x}[n]=\left[x_{1}[n], x_{2}[n], \cdots, x_{N_{T}}[n]\right]^{T}, \mathbf{y}[n]=\left[y_{1}[n]\right.$, $\left.y_{2}[n], \cdots, y_{N_{R}}[n]\right]^{T}$ and $\mathbf{H}[\mathbf{n}]$, is a matrix with $h_{i k} e^{j 2 \pi \Delta f_{i k} n T_{S}}$ as its elements. In the steady state, knowing all $h_{i k}$ and $\Delta f_{i k}$, $\mathbf{y}[n]$ can be solved using the pseudo-inverse of $\mathbf{H}$ by standard zero forcing technique, as long as $N_{R} \geq N_{T}$, which minimizes the least square error as,

$$
\tilde{\mathbf{x}}[n]=\left(\mathbf{H}^{\top} \mathbf{H}\right)^{-1} \mathbf{H}^{\top} \mathbf{y}[n]
$$

The above technique of decoding is known as zero forcing [36]. In some cases $\mathbf{H}$ may be ill-conditioned (low rank) and the pseudo-inverse maybe very sensitive to noise. To avoid sensitivity to noise Minimum Mean Square Error (MMSE) [36] uses the equation,

$$
\tilde{\mathbf{x}}[n]=\left(\mathbf{H}^{\top} \mathbf{H}-\mathbf{N}\right)^{-1} \mathbf{H}^{\top} \mathbf{y}[n]
$$

In Eqn $4, \mathrm{~N}$ is a diagonal matrix with $N_{i i}$ is the noise floor value of the receiver $i$.

\subsection{Blind Estimation}

When $\mathcal{K}$ new packets arrive (such as $P_{1}$ and $P_{2}$ in Fig. $12(\mathrm{c})$ ) with $N_{T}$ concurrent transmissions in previous steady state phase, Eqn 1 can be rewritten as:

$$
\begin{aligned}
y_{i}[n]= & \sum_{k}^{N_{T}} h_{i k} \cdot e^{j 2 \pi \Delta f_{i k} n T_{S}} \cdot x_{k}[n]+ \\
& \sum_{l}^{\mathcal{K}} h_{i l} \cdot e^{i 2 \pi \Delta f_{i l} n T_{S}} \cdot P\left[n-n_{l}\right]
\end{aligned}
$$

where $P[n]$ are the known preamble samples, and $n_{l}$ is the arrival time of each packet, with $n_{1}$ being the earliest one. $h_{i k}, f_{i k}$ have all been estimated for $i=1 \cdots N_{T}$ at each receiver $j$. Thus, we need to jointly decode the following unknown variables. (i) The samples from $N_{T}$ concurrent transmissions: $x_{i}[n], \forall i=1 \cdots N_{T}$; (ii) $\mathcal{K}$ New packets' channel and CFO for each receiver: $h_{i l}, f_{i l}, \forall l=1 \cdots \mathcal{K}, i=1 \cdots N_{R}$.

We adopt the idea provided in [58] to reduce the unknown number of CFOs from $\mathcal{K} N_{R}$ to $\mathcal{K}$. The idea relies on the observation that the CFOs among base-stations remain constant for several seconds and can be measured periodically. Thus, knowing CFO to any single BS, allows us to derive the same for the remaining BS. Let the CFO among two basestations $i$ and $j$ as $\delta_{i j}$ be known. Therefore, Iris only needs to estimate one CFO variable between transmitter $k$ and a reference receiver $r$, which is $\Delta f_{r k}$. The rest CFO values can be obtained as $\Delta f_{j k}=\Delta f_{r k}+\delta_{r j}$. Utilizing the known clock offsets among receivers reduce the number of CFO variables from $N_{R}$ to 1 for each transmitter.

The known preambles provide an opportunity to decode all these unknowns. With $M$ samples, we accumulate $N_{R} \cdot M$ equations, and try to solve $N_{T} \cdot M$ data samples variables, $\mathcal{K}$ $\mathrm{CFO}$ and $\mathcal{K} N_{R}$ channel variables. Our goal is to estimate the unknowns by solving the over-determined set of equations in a least mean square sense.

A key challenge in solving the equations formed by Eqn 5 is that the set of equations are non-linear due to the CFO terms which are multiplied with the unknown channel variables. We arrange the equations in Eq 5 into the form of $\mathrm{Ax}=\mathrm{b}$, where $\mathbf{x}$ represents the vector containing all the unknowns: $\mathcal{K} N_{R}$ channel variables $\left(h_{j k}, k=1 \cdots \mathcal{K}, j=\right.$ $\left.1 \cdots N_{R}\right)$ and $N_{T} \cdot M$ sample variables $\left(x_{i}[n], i=1 \cdots N_{T}\right)$. A represents the coefficients, which contains the $\mathrm{CFO}$ values. Treating the $\mathrm{CFO}$ values as known values, we use least square approach to solve the over-determined linear equations, with the residual error as $\|\mathbf{A} \tilde{\mathbf{x}}-\mathbf{b}\|^{2}$, where $\tilde{\mathbf{x}}=\left(\mathbf{A}^{\top} \mathbf{A}\right)^{-1} \mathbf{A}^{\top} \mathbf{b}$ (Eqn 4 for MMSE). Therefore, we formulate the following optimization problem to estimate CFO and solve for data samples concurrently.

$$
\begin{array}{ll}
\underset{\Delta f_{r k}, \forall k=1 \cdots \mathcal{K}}{\operatorname{minimize}} & \|\mathrm{A} \tilde{\mathbf{x}}-\mathbf{b}\|^{2} \\
\text { subject to } & \tilde{\mathbf{x}}=\left(\mathbf{A}^{\top} \mathbf{A}\right)^{-1} \mathbf{A}^{\top} \mathbf{b}, \\
& \Delta f_{j k}=\Delta f_{r k}+\delta_{r j}, \\
& \forall j=1 \cdots N_{R}, j \neq k, \forall k=1 \cdots \mathcal{K} .
\end{array}
$$

In our implementation we use the Nelder-Mead Optimization [53] for the above non-linear optimization.

\subsection{Conditions for Blind Estimation}

After $M T_{S}$ time in the blind estimation state, Iris has $M N_{R}$ equations corresponding to $M$ received IQ samples at each of the $N_{R}$ receivers. There are $M N_{T}$ number of unknown data samples to be estimated for the $N_{T}$ ongoing transmissions from the previous steady state phase, $N_{R} \mathcal{K}$ unknown channels and $N_{R}$ CFOs for the $\mathcal{K}$ new transmissions. Thus, in order to solve for the unknowns,

$$
M N_{R} \geq M N_{T}+\left(N_{R}+1\right) \mathcal{K}
$$

We name Eqn 6 the Blind Estimation Sufficiency Condition (BESC). As shown in Fig. 11, the decoding transfer to steady decoding if BESC can be satisfied ${ }^{1}$.

\footnotetext{
${ }^{1}$ Note that Eqn 6 is the worst case since if some transmissions end earlier then the number of unestimated data samples will be less than $M N_{T}$
} 
Duration of Blind Estimation Phase. In order for Eqn 6 to be satisfied the blind estimation phase must last at least $M$ samples, where

$$
M_{\min } \geq \frac{\left(N_{R}+1\right) \mathcal{K}}{N_{T}-N_{R}}
$$

Minimum Number of Receivers Needed. From Eqn 7 it is clear that $N_{R}>N_{T}$, otherwise $\tau_{0}$ becomes unbounded. Note blind estimation requires one additional receiver than traditional MIMO, where $N_{R} \geq N_{T}$.

Burst Limit Condition. Eqn 6 assumes that the IQ samples from the $\mathcal{K}$ transmissions are known since they correspond to the preambles. If the blind estimation phase lasts longer than the duration of preamble length $L_{P}$ in samples, i.e. $M_{\min }>L_{P}$, then the above assumption will no longer be true. Under this condition, the transmissions whose channels have not yet be estimated will add to the unknown data samples and the number of unknown data symbols will grow faster than the number of equations. Thus, for blind estimation to work, the burst size should be limited to,

$$
\mathcal{K}_{\text {max }}=\mathcal{K} \leq \frac{\left(N_{R}-N_{T}\right) L_{P}}{N_{R}+1}
$$

In Sec. 4.5 we discuss how Iris recovers and functions under scenarios where the burst limit condition is violated.

\subsection{Handling Error Conditions}

In this section we discuss the various error conditions Iris might experience and how it recovers from them.

Preamble Detection Errors. There can in general be three kinds of preamble detection errors - missed detection, where a preamble is completely missed, incorrect start of frame, where the starting point of the payload is incorrect and false detection, where a preamble is detected when there was no transmission. Given that preambles are usually designed for robust detection, the first two errors usually occur when the transmission is too weak compared to the others. Such weak transmissions are usually too weak to "significantly" degrade the decoding of the other transmissions. In case of a falsely detected preamble, the estimated channel amplitude is usually very small since there was no preamble in the first place and thus ends of not having a significant effect on the decoding process as well. While we have observed and simulated these in practice, we do not provide the results in the paper due to lack of space.

Traffic Burst Exceeds Burst Limit When several devices begin transmitting within a very short time, traffic bursts occur. If either $N_{T} \geq N_{R}$ or $\mathcal{K}>\mathcal{K}_{\max }$ (Eqn 8) Iris will not be able estimate the IQ samples and decode packets. Iris can detect if any of the above conditions are violated while trying to decode and take corrective action if necessary. The recovery of Iris from a large burst depends on whether the underlying access is slotted (devices are time synchronized and their transmissions respect slot boundaries) or unslotted. We now discuss each of these scenarios.

Recovery From Bursts in Slotted Access : If IoT multiple devices transmit at exactly the same time (within $\pm 80 \mu \mathrm{s}$ ), Iris will not be able to distinguish their preambles and hence will not be able to decode them separately. This can be avoided by making the IoT devices adding a small (few ms) random delay before their transmissions. In circumstances where, the burst limit is exceeded in a certain slot, all the packets will be lost. However, Iris returns to the idle state at the end of each slot period and so the effect of burst in the previous slot is not carried over to the next slot.

Recovery From Bursts in Unslotted Access : In the absence of slot boundaries, inability to decode packets can get carried over to successive overlapping packets leading to a cascade of failures until, Iris returns to the idle state. While this will occur naturally as soon as the burst dissipates, it may be more efficient for the base-station to explicitly signal the devices (e.g. through a busy tone) to stall transmitting for a short period of time (usually two packet transmission intervals or about $100 \mathrm{~ms}$ ). This will allow Iris to return to idle state and resume as usual.

\section{IMPLEMENTATION AND DEPLOYMENT}

Implementation of Iris requires access to raw IQ samples from the radio. Commercial MURS band radios e.g. Raveon RV-M7-GX do not provide this capability. In addition, Raveon devices use proprietary preambles, compression and encryption mechanisms that we do not have access to. Consequently, we used USRP (B200, N210) for our implementation of Iris with WBX-40 radio daughterboards.

Narrowband channelization. The USRP radio daughterbaords are not inherently designed to support narrowband $12.5 \mathrm{kHz}$ channels. WBX-40 has $40 \mathrm{MHz}$ bandwidth. USRP supports reducing this bandwidth to $200 \mathrm{kHz}$ through digital filtering on the on-board FPGA. In order to reduce this bandwidth to $12.5 \mathrm{kHz}$, we used a digital elliptic lowpass filter with a passband of $12.5 \mathrm{kHz}$ and $50 \mathrm{~dB}$ rejection. The filtered signal was downsampled to $12.5 \mathrm{k}$ samples $/ \mathrm{sec}$.

Bandwidth required per channel. Each raw IQ sample comprises two 16 bits values one each for I and Q components. At $12.5 \mathrm{k}$ samples/sec, the total bandwidth generated is $400 \mathrm{kbps}$. This is then transmitted to a central entity in the cloud for joint decoding using Iris.

Packet structure and modulation. We used FSK modulation with $2.5 \mathrm{kHz}$ deviation as specified by FCC MURS regulations. We used Barker codes for preambles to improve preamble detection. The payload bits were randomly generated, and each packet is $50 \mathrm{~ms}$ long. We use the GFSK module in GNURadio [8] for modulation and demodulation. 
IoT devices. We use USRP B200 as IoT transmitters, which have maximum power output of $10 \mathrm{dBm}$. In order to allow these devices to transmit at $2 \mathrm{~W}$, we used the ZHL-1-2W-S+ power amplifier from Mini-Circuits [1].

Base-stations. Our current deployment uses USRP N210 with WBX-40 daughterboard as four base-stations, with two each placed on two roof-tops of $20 \mathrm{~m}$ high buildings located $1.3 \mathrm{Km}$ apart (shown in Fig. 14). Each base-station is equipped with a single omnidirectional antenna [6].

Implementation of Iris. Iris has been implemented in C and Python. The steady state decoding and CFO optimization are coded $\mathrm{C}$ in a multi-threaded fashion as described in Sec. 6.2. We use GNU Scientific Library [3] for parallel matrix inverse, and a non-linear optimization library NLopt [7] to implement Nelder-Mead method. As discussed in Sec. 4.2, both zero forcing and MMSE can be used for decoding. We implemented both techniques and observe no notable difference in performance. The results in Sec. 6 are obtained with zero forcing.

Limitations of using USRP. As discussed earlier USRP radios are not designed specifically for $12.5 \mathrm{kHz}$ MURS band like the RV-M7-GX radios. Consequently, even though we use digital filters in our implementation to transmit and receive on MURS band channels, our USRP devices suffer from a significantly higher noise floor compared to the RVM7-GX radios. Further, we do not use any forward error correction (FEC) coding unlike RV-M7-GX radios. These factors together contribute to reduce the range of our USRP deployment to $\approx 3 \mathrm{~km}$, significantly lower compared to 13 14Km using RV-M7-GX.

\section{EVALUATION}

In evaluating Iris we ask three key questions,

- How well does Iris decode concurrent uncoordinated long rage uplink transmissions in scenarios where nonMIMO techniques fail? In order to answer this question we use our Iris deployment (Sec. 5) to perform outdoor tests.

- How much gains in network capacity can Iris provide for a real operational IoT network deployment compared to non-MIMO collision avoidance and resolution schemes? Since our USRP deployment does not provide the necessary scale to answer this question, we perform trace driven simulations on traces from our 90 vehicle operational IoT network.

- How does Iris scale to a large number of base-stations for computation in the cloud? We use our implementation in the cloud and benchmark the running time of Iris and describe how Iris is amenable to parallel execution.

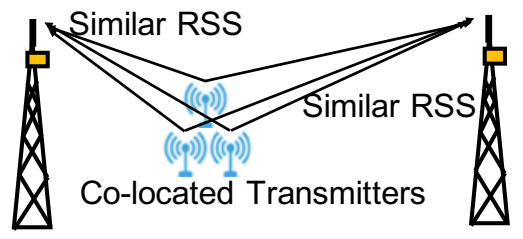

Figure 13: Co-located transmitters having similar RSS.

\subsection{Outdoor Testing of Iris}

To demonstrate the effectiveness of Iris we first test the hardest scenario to decode concurrent transmissions - when all the transmissions originate from a set of very proximate locations (all IoT devices mounted on the same car) as depicted in Fig. 13. This scenario is particularly hard since, at any given base-station all concurrent transmissions will be received at almost the same RSS. This means that techniques such as successive interference cancellation (SIC) that leverage the difference in RSS between concurrent transmissions to iteratively decode the stronger signals are expected to fail. Further, diversity based techniques that leverage the fact that the relative RSS ordering of different transmissions might be different will also not perform well. Next, in order to investigate how well Iris works when RSS are different, we control the power levels of the different transmitting devices to achieve a variety of RSS differences.

Experiment Description. We place three transmitters in a single car. The transmissions are received at four basestations, with two each placed on two roof-tops of $20 \mathrm{~m}$ high buildings located $1.3 \mathrm{~km}$ apart (shown in Fig. 14). Each base-station is equipped with a single omnidirectional antenna. We drove the car around stopping at locations where we could park to conduct the experiment at 17 different locations shown in Fig. 14. At each location, we control the transmitters to create three different scenarios with collision free, 2 and, 3 concurrent transmissions. Three B200 are connected to a host laptop via USB3, which can be coarsely synchronized down to a few tens of samples. Such control is sufficient to induce intentional 2 and 3 packet collisions. For each experiment the transmitters transmit 126 packets.

Other collision resolution schemes for comparison. In addition to Iris we also attempt to decode the packets using four other schemes. First, in order to measure SNR of the individual transmissions we transmit from each of the three devices individually deemed Collision-free. Our Baseline scheme comprises decoding plainly without any collision resolution. Antenna Diversity relies on the chance that given different wireless channels, it maybe possible that at one of the antennas, a transmission might be received at a high power and be amenable to decoding. In the SIC scheme, SIC is applied individually at each base-station attempting to decode as many packets as possible. 


\section{$3.1 \mathrm{~km}$}

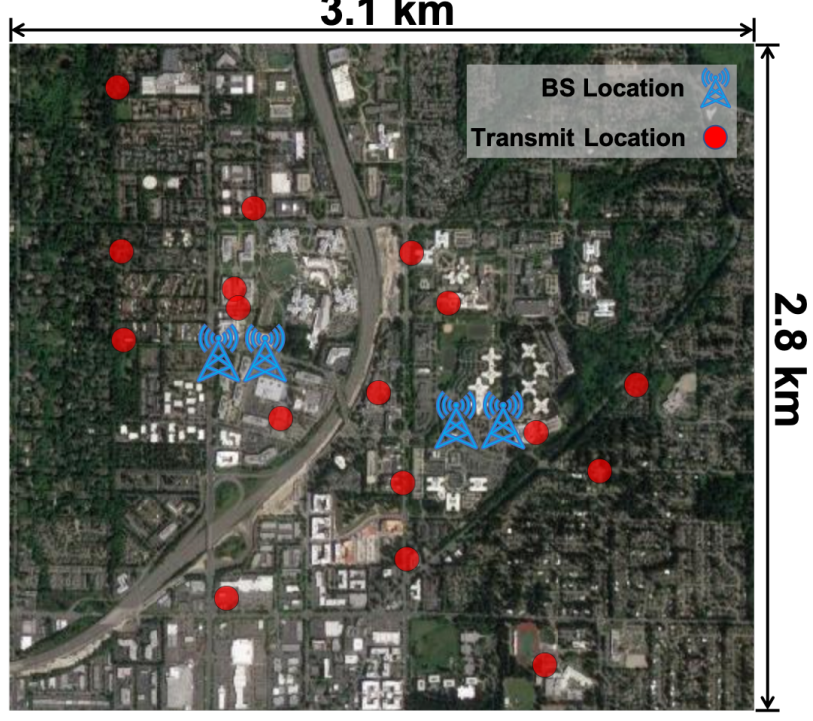

Figure 14: BS deployment and transmit locations. Two BS are placed at each location.

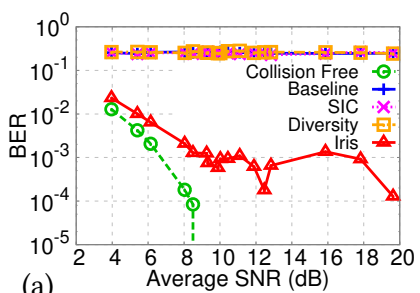

(a)

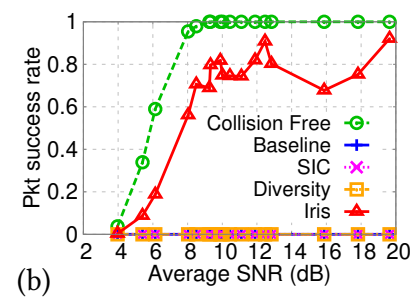

Figure 15: Decoding accuracy at different locations. (a) Average BER. (b) Average success packet rate.

Decoding Accuracy vs Average SNR. In our experiment, each transmission has four SNR values corresponding to the four different receiving base-stations. The decoding accuracy is expected to depend on all the four SNR values. Fig. 15 (a) and (b) depict Iris's decoding bit error rate and average packet success rate versus the average SNR across the four base-stations and compare it against the other schemes at each location. The corresponding curves for collision-free scenarios is also provided for reference. As expected, none of the schemes other than Iris is able to decode transmissions even at SNRs as high as 20dB. This is because as discussed, in our experiment, at each individual antenna each transmission experiences interference from one or two other equally strong transmissions.

Fig. 16 plots the BER and packet success rate for Iris's decoding, under 2 and 3 collision cases. Not surprisingly, slightly better performance is achieved with fewer collisions,
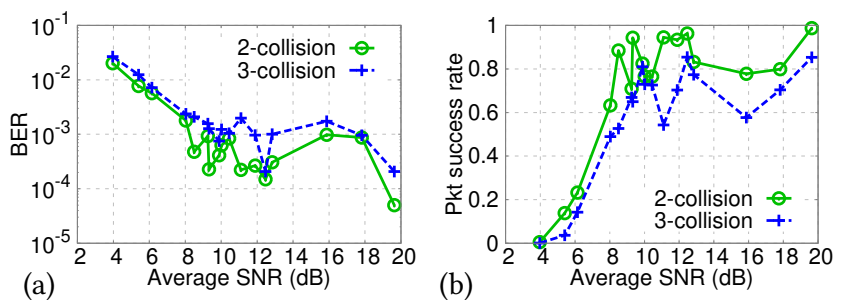

Figure 16: Iris's decoding accuracy under 2-collision and 3-collision cases. (a) Average BER. (b) Average packet success rate.
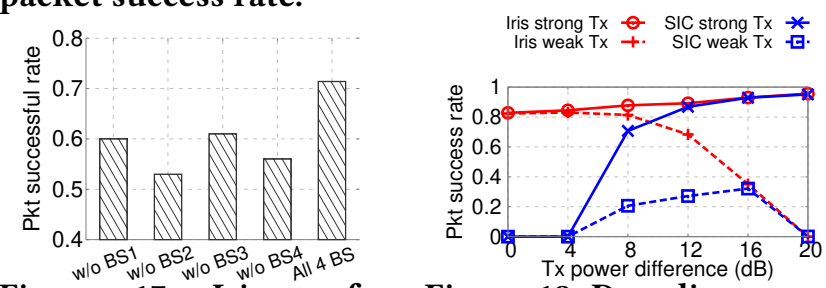

Figure 17: Iris perfor- Figure 18: Decoding accumance using a subset of racy with different transreceivers. mit power.

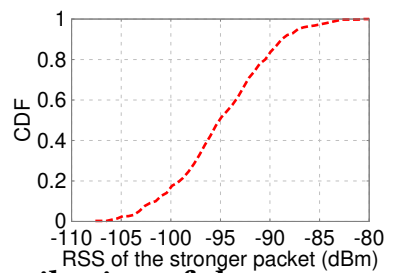

Figure 19: Distribution of the stronger packet's RSS.

which makes the decoding problem more over-determined (Sec. 4.2 and 4.3).

Does using more base-stations help? We now ask the question, "For the same number of concurrent transmissions, does using a greater number of base-stations improve performance?" As discussed in Sec. 4, having more receivers helps create an over-determined system, improving performance in general. To validate this argument, we focus on 2-collision scenarios, which requires a minimum of 3 receivers to decode the packets. With a total of 4 base-stations in all, there are 4 possible distinct subsets of three base-stations created by removing one of the BS. For each of the four subsets we decode using Iris using only the three base-stations in the subset. Fig. 17 depicts the overall packet success rate over all the locations for each of the four subsets as well as that by using all four base-stations for 2 and 3 collision scenarios. As seen from Fig. 17, choosing any specific subset of three base-stations yields in poor overall performance compared to using all 4 in Fig. 17.

SIC versus Iris when RSS are different. In all prior experiments, colliding transmissions were received at the same power at every base-station and thus SIC was unable to decode. However, SIC's performance is expected to improve as the received transmissions have different RSS values. In order 

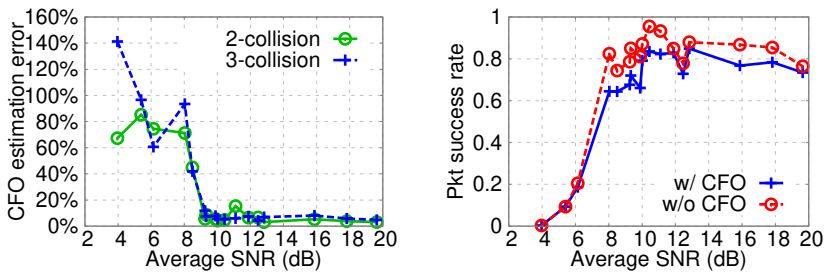

Figure 20: CFO estimation Figure 21: Impact of CFO error. error.

to understand how SIC compares to Iris as the difference in received RSS increases, at each location we create 2-collision transmissions with one device transmitting at lower power. We conduct the same experiment at several different steps of lower power levels. Fig. 18 plots Iris and SIC's packet success rate, for both the stronger and weaker transmitter.

As seen from Fig. 18, Iris decodes both strong and weak transmissions well up to $12 \mathrm{~dB}$ RSS difference between the stronger and weaker signals, however, it is unable to decode when the difference is $20 \mathrm{~dB}$. Not surprisingly SIC is unable to deode either the strong/weak transmissions until about $8 \mathrm{~dB}$ difference between the stronger and weaker signals. As this difference increases, the stronger signal is received reliably. It is surprising to note however that the weaker transmission's reception gains only until $40 \%$ at $16 \mathrm{~dB}$ difference and falls to almost zero when the difference is $20 \mathrm{~dB}$. This is unexpected since, the SIC should be able to cancel the stronger signal perfectly and recover the weaker signal at $20 \mathrm{~dB}$ SINR. The reason for this is revealed in Fig. 19 which depicts the CDF of the stronger transmissions' RSS. As seen from Fig.19, over $98 \%$ of the RSS are below $-85 \mathrm{dBm}$ and over $80 \%$ are below $-90 \mathrm{dBm}$. At $20 \mathrm{~dB}$ difference, the weaker signal is below $105 \mathrm{dBm}$ over $98 \%$ of the time and below $-110 \mathrm{dBm}$ over $80 \%$ of the time. Given that $-105 \mathrm{dBm}$ is the practical decoding limit for the transmissions, the weaker transmission is not decodable either by SIC or Iris. This is distribution of RSS is typical in practical deployments as well. For example as seen from Fig. 5, RSS flls to below $-90 \mathrm{dBm}$ at a distance of $3 \mathrm{Km}$ while the range is about $14 \mathrm{Km}$. This means that for over $95 \%\left(100 \frac{14^{2}-9^{2}}{14^{2}}\right)$ of the coverage area, the RSS will be below $-90 \mathrm{dBm}$.

CFO estimation error and its impact. A key operation in the blind estimation phase is CFO estimation via an optimization process (Sec. 4.3). To provide insight into how accurately Iris estimates the $\mathrm{CFO}$ and the estimation error's impact, we use the collision free reference packets to obtain a ground truth of the CFO, and compare Iris's estimated values against them.

Fig. 20 plots the $\mathrm{CFO}$ estimation error for different SNR values. As SNR improves to $10 \mathrm{~dB}$, the estimation error quickly drops below 5\% in most cases for both 2-collision and 3collision, showing the effectiveness of Iris's optimization process. To understand the effect of CFO estimation error on packet success rate, Fig. 21 compares packet success rates obtained by using the groundtruth $\mathrm{CFO}$ (w/o CFO) versus using the CFO estimated in blind estimation (w/ CFO). At low SNR, correcting CFO error does not improve PSR significantly indicating that noise, rather than CFO error, is the primary cause for low decoding accuracy in this case. At high SNR values ( $>10 \mathrm{~dB})$, the impact of CFO errors increases and there is reduction of about $10 \%$ in packet success rate due $\mathrm{CFO}$ estimation errors.

\subsection{Real Time Decoding Latency}

Iris decoding occurs in the cloud. In order to keep up decoding in real time and scale to a large number of base-stations, Iris's implementation must be amenable to parallel execution so that it can be scaled across multiple cores. In this section we benchmark how Iris scales to support real time decoding for a large number of base-stations making using of multiple cores in the cloud.

Iris is amenable to two kinds of parallelization. First in steady state each sample period can be decoded independently in a separate instance since there is no dependency across sample periods. Second, within a single sample period, the matrix inversion as well as optimization libraries used in steady state and blind estimation are amenable to multithreaded with our implementation introduced in Sec. 5.

To evaluate Iris's operation latency, we perform decoding operation on a 10 core Intel Xeon E5-2630 processor, with and without enabling parallelization. The decoding latency under different number of concurrent transmissions is plotted in Fig. 22. With $4800 \mathrm{bps}$, a 32 -byte packet is $53.3 \mathrm{~ms}$ long. Iris is guaranteed to keep up with incoming packets as long as the decoding time for each set of concurrent packets is less than $53.3 \mathrm{~ms}$, which is plotted as the green dotted line in the figure. Clearly, the decoding latency is unacceptable without enabling parallel processing. On the other hand, Iris can process up to 30 concurrent packets at real time. Reflecting on a real-world network, 14 2-antenna base-stations can support 30 concurrent transmissions, which are sufficient to provide coverage for several cities. For different geographical regions that are far apart, one can instantiate a separate instances of Iris since they are expected to have no common receiving base stations and scale to entire states or an entire country.

\subsection{Large Scale Trace Driven Simulations}

In this section we ask the question "How much network capacity gains can Iris provide in a real large scale IoT network deployment?" In order to answer this question we leverage 

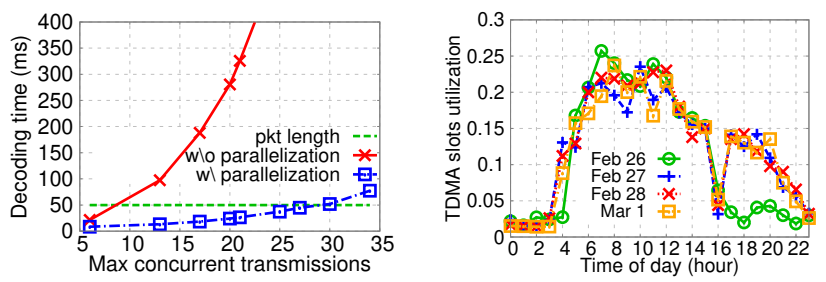

Figure 22: Decoding la- Figure 23: TDMA Utilizatency. tion for our 90 vehicle pilot
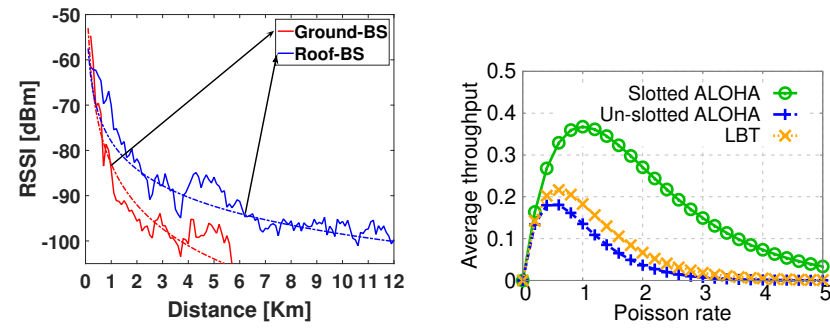

Figure 24: Ground- Figure 25: Ineffectiveness ground channel versus of CSMA ground rooftop channel

the network traffic traces from our 90 vehicle deployment (described in Sec. 2). Our currently deployed network vehicles transmit every 10s, over preassigned TDMA slots to avoid collisions. However, since vehicles transmit messages only when they are moving, only a fraction of these slots are used at any given time. Fig. 23 depicts the utilization of number of TDMA slots as a function of hour of day in our current network for 4 consecutive days, Tuesday-Friday. As seen from Fig. 23, even during peak hours the utilization is below 25\% indicating the oversubscribed nature of TDMA.

Methodology for Simulations. In our simulations, we use a hexagonal cellular deployment. The distance between BS (cell centers) is chosen to be the distance where mean RSS falls $-100 \mathrm{dBm}$. Based on the path loss curve obtained from our deployment in Sec. 2, this distance is $8 \mathrm{~km}$.

Our current IoT network uses TDMA and hence using the traces themselves as is will yield no collisions. In the absence of TDMA however, while each vehicle would still continue to transmit periodically once every 10 seconds, the offset time of transmission within the 10 second epoch would be determined by when the vehicle first started moving i.e a random number between 0 to 10 . In order to emulate this behavior, we slightly alter the trace, by offsetting the start time of each vehicle's move segment (a continuous period that the vehicle keeps moving) by a random amount, in the range of 0 to 10 seconds.

Our network today operates well below its capacity, but we want to ask the question "what would happen if we used more vehicles and what would be the network capacity for different schemes?" To increase the number of vehicles, while preserving the underlying traffic characteristics we superimpose multiple days diurnal traffic traces into one. When superimposing, instances of the same vehicle from different days are considered to be different unique transmitters. In order to compute the RSS at the base-stations we used the actual GPS locations of the vehicles from the traces but used the path loss model obtained from the measurements in Sec. 2. In order to estimate the log-normal fading in the channel we measured the standard deviation in RSS for receptions of the same transmission at two BS antennas separated by $10 \mathrm{~m}$ over 300,000 transmissions and found this to be $3.6 \mathrm{~dB}$.

Ineffectiveness of Listen Before Transmit (LBT). While LBT is not mandated in MURS bands, it is extensively used in WiFi to avoid collisions. We ask the question, how effective is LBT for long range IoT networks? Long range IoT networks differ from indoor LANs is that while devices are at the ground level, the base-station itself is located high up on a roof top. Consequently, the signal propagation between devices at the ground level experiences significantly higher number of obstacles and hence, a much greater path loss than that between the device and the base-station. Base-stations are also equipped with higher gain antenna that the IoT devices due to form factor and cost constraints.

Fig. 24 captures the significant difference in range for ground-ground versus and ground to roof BS. In this experiment, we deployed a device located at the ground level $(\approx 2 \mathrm{~m}$ high on top of a parked car) that was configured to receive similar to a BS. The MURS base-station, itself was on the roof $20 \mathrm{~m}$ high building right across the street from this device. We then placed a second device in another car and drove around obtaining over 40,000 measurements in an urban environment at various distances. Thus, transmissions were received at both the static device and the base-station from the same set of locations. Fig. 24 shows that the range of the ground-ground channel is $\approx 5.5 \mathrm{~km}$ while the same for the ground-roof channel is $\approx 14 \mathrm{~km}-2.5 \times$ greater, based on using $-105 \mathrm{dBm}$ as the range.

With a carrier sensing range of $-100 \mathrm{dBm}$ (recommended by manufacturer), an IoT device has a sensing range of $3.5 \mathrm{Km}$ - $\approx 4 \times$ less than the range of the base-station. In other words, devices at the ground level will not be able to sense $\approx 93 \%$ $\left(1-\frac{1}{4^{2}}\right)$ of the other transmitting devices within range of the base-station (hidden terminals). Fig. 25 compares the theoretical normalized throughout of LBT with $93 \%$ hidden nodes with ALOHA and slotted ALOHA. As seen from Fig. 25 LBT performs almost as well as un-slotted ALOHA.

Simulation Results. Fig. 26 (a), plots the fraction of successfully received packets over an entire day from all those that were transmitted for different number of vehicles when each base-station has one antenna. As the number of vehicles increases, the number of concurrent transmission increases 


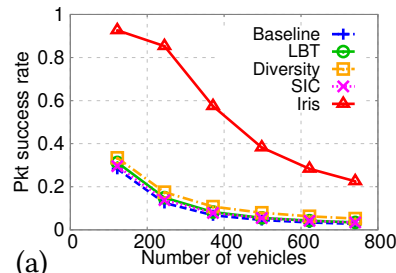

Figure 26: Throughput under different number of vehicles. (a) Each base-station has one antenna; (b) Iris with different number of antennas.

as well, resulting in more collisions. As seen from Fig. 26 (a), SIC performs best among existing approaches and is able to achieve about $25 \%$ reception for 100 vehicles, however this performance does not improve significantly with more antenna at each base-station. Iris, on the other hand, with only one antenna at each base-station achieves around $3 \times$ throughput over SIC for 100 vehicles and $5 \times$ for 200 vehicles. This demonstrates how distributed MIMO using multiple base-stations (one in each of the 7 neighboring cells) helps decode concurrent transmissions. Fig. 26 (b), examines the effect of deploying more (2-4) antennae at each base-station. 1-4 antennae per base-station. As seen from Fig. 26 (b), capacity scales (almost linearly) with an increase in the number of antennae/BS and with 4 antenna, about 800 vehicles can be supported.

\subsection{Analysis of Traffic Bursts}

In this section, we use a packet level simulations to understand how traffic bursts effect Iris and how it recovers from when sudden large bursts hamper its decoding. As discussed in Sec. 4.3 and 4.4, Iris cannot decode if either the number of transmissions is greater than the number of receivers or if the burst limit is violated. In this section through simulations, we analyze how such conditions effect overall capacity. Since analysis is not analytically tractable, we conduct packet level simulations with packet arrivals following a Poisson process. Packets are lost only under bursts that disallow Iris's decoding. All packets in such a burst are assumed to be lost. We implement slotted, unslotted and unslotted with busy tone feedback (as discussed in Sec. 4.4) to dissipate the bursts. There are no downlink acknowledgements.

As expected the general trend in all capacity curves Fig. 27 and 28 is that the capacity first increases with Poisson rate as the channel utilization grows higher and drops as the high rate causes lost packets due to large bursts that Iris is unable to decode. The maximum capacity corresponds to the peak capacity in the curves. To provide a baseline when Iris is not used, since the packet duration is $50 \mathrm{~ms}$, a maximum throughput of 20 packets/sec is possible in the absence of MIMO. The maximum theoretical capacity for slotted and unslotted access corresponds to 7.4 packet/sec $\left(\frac{20}{e}\right)$ and 3.7
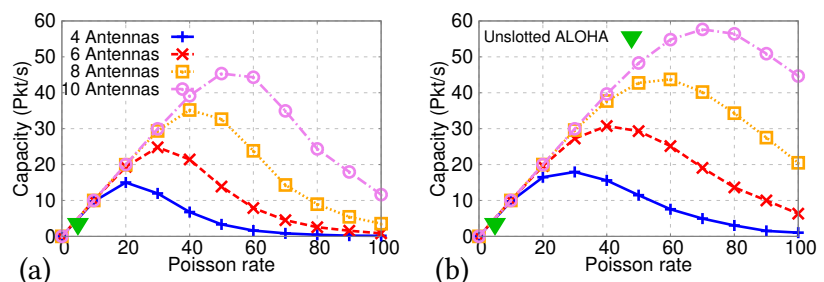

Figure 27: Unslotted access network capacity with different number of antennas (a) without busy tone (b) with busy tone. Green marker represents optimal unslotted ALOHA.
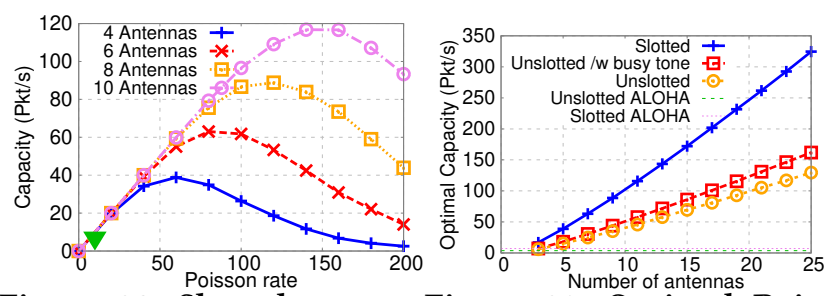

Figure 28: Slotted access Figure 29: Optimal Poisnetwork capacity. Green son capacity of Iris. marker indicates optimal slotted ALOHA.

packets/sec $\left(\frac{20}{2 e}\right)$ respectively. Fig. 29 depicts that maximum capacity of the network as a function of number of receiving antennae for slotted, unslotted and unslotted with busy tone respectively. As seen from Fig. 29, the capacity scales linearly with increase in the number of antennae and as expected slotted access provides little over $2 \times$ the capacity of unslotted access. The use of busy tone to signal to occurrence of a burst in unslotted access improves the capacity by about $10 \%$.

\section{RELATED WORK}

Low-Power Wide-Area Networks. LPWAN has gained increasing interest over recent years. Semtech [4] and SigFox [9] have developed chipsets to empower this technology with narrow bands of unlicensed spectrum. Over licensed band, LTE-M [60] and NB-IOT [59] standards have been developed for cellular base stations to communicate with low-power IoT devices. LPWAN's range and performance have been analyzed and measured in [51, 57, 70]. More recent studies have focused on improving LPWAN's scalability [33], range $[31,32]$, and energy efficiency $[55,68,73]$.

Distributed MIMO. Distributed MIMO systems synchronize distributed transmitters to enable them to concurrently transmit to multiple independent receivers, without interference. It has been studied in the contexts of sensor networks [29], wireless LANs [30] and cellular networks [46]. In the past, a number of works have shown from a theoretical perspective how distributed MIMO improves throughput as the network size grows [15, 49, 65]. More recently, distributed MIMO has been moved from theory to practice 
$[14,18,21,39,40,58,79]$, demonstrating the ability to harvest both diversity and multiplexing gain. Differ in details, these works focus on a variety of problems, including synchronizing the transmitters in time, phase and frequency, power control, and controlling overhead of channel measurements. Successive Interference Cancellation. The achievable capacity of SIC in various types of networks have been derived and analyzed $[71,72,75]$. The emergence of software-defined radios makes it possible to implement this technique in practice, and a number of works have shown SIC's ability to improve link layer performance [16, 45, 54, 76]. In general, SIC's performance depends on whether the current strongest signal has sufficient SNR in the combined signal. A number of works have shown that SIC is only able to achieve decent performance when different signals' power levels meet certain requirements $[63,64]$.

Non-orthogonal multiple access (NOMA) In contrasts to conventional orthogonal multiple access, NOMA shares the same radio resources among users at the cost of increased interference [27, 61]. Recent power domain NOMA primarily relies on SIC in uplink for the base-station to decode concurrent messages, which can be aided with power control to improve performance [43, 67]. Power control over uplink requires channel information from clients to the basestations, which requires coordinated measurements. Without any power control, uplink NOMA performance highly depends on the effectiveness of SIC. Particularly, the channel (power) of different transmitters need to be distinct for SIC to perform well [43].

Blind MIMO decoding. The idea of blind MIMO decoding has been proposed before. Talwar et al. apply MMSE techniques to decode over small alphabets while simultaneously recovering the channel gain matrix [69]. Authors in [41] present a statistical learning algorithm that uses the estimate of the covariance matrix of the received signals to learn the channel gain matrix. More recent works have significantly improves the performance and efficiency $[28,56]$. However, exisiting blind estimation approaches typically rely on special structures in the modulation. For example, the design in [41] requires the modulation constellation to be discrete, and needs a large number of samples to decode, while [28, 56, 69] only work with hypercubic constellations. such as BPSK and MPAM.

Cloud RAN. Iris's design largely falls in the domain of cloud radio access network (RAN), an emerging mobile network architecture which can address a number of challenges the operators face while trying to support growing end userâĂŹs needs. It was first proposed in [47]. The main idea is to collect the baseband signals from multiple base stations into centralized pool for coherent combining [24, 25, 38, 77]. Cloud RAN is targeted by a number of mobile network operators worldwide, including , IBM [47], Alcatel-Lucent [62], Nokia
Siemens Networks [37], Intel [23] and Texas Instruments [34]. It is also a candidate for future generation of cellular architecture [26]. Recent researches have focused on adapting to nonuniform traffic and scalability [22, 48, 50, 52], reducing energy and cost [44], and improving throughput, delay performance $[42,46,78]$. These past researches are mostly in the cellular network realm.

Most similar to Iris, OpenChirp [31] and Charm [32] propose to allow multiple LoRaWAN gateways to pool their received signals in the cloud, coherently combining them to detect weak signals. In the authors proposal, multiple gateways utilize LoRa's modulation scheme to detect weak signals, and send the packet raw IQ samples to the cloud. Through effective grouping and time synchronization algorithms at the cloud, Charm jointly decodes with coherent MIMO combining. Charm's ability to decode weak signals significantly improves LPWAN's range and client device battery life. Iris, on ther other hand, focuses on a different problem, handling packet collisions in long range LPWAN.

\section{CONCLUSIONS}

In this paper we show that the VHF narrowband channels including unlicensed MURS bands as well as the licensed narrowband channels can offer far superior geographical coverage compared to ISM bands for deploying long range wide area IoT networks. This increased geographical coverage can help cover large areas at a significantly smaller infrastructure deployment and management costs. We prove this through a large scale deployment over 400 sq Km involving 90 vehicles. In order to meet the increased capacity demands arising out of a greater number of IoT devices, we propose a novel distributed multi-user MIMO technique Iris, that does not require any coordinated measurements between IoT devices and base-stations. This is inherently well suited for IoT deployments since IoT devices typically transmit messages in response to unpredictable sporadic events and prefer to sleep during other times. Through field deployments and trace driven simulations based on our IoT, we demonstrate that Iris is practically viable and can offer $4 \times$ capacity gains for each antenna added at each base-station.

\section{ACKNOWLEDGEMENTS}

We would like to thank all our shepherd Hun-Seok Kim and anonymous reviewers for their comments that helped us in improving the quality of the paper. This research was supported in part by US National Science Foundation through CNS-1838733, CNS-1719336, CNS-1647152, and CNS-1629833. 


\section{REFERENCES}

[1] [n.d.]. Amplifier for Client Devices. https://www.minicircuits.com/ WebStore/dashboard.html?model=ZHL-42W\%2B

[2] [n.d.]. FCC Narrowbanding Overview. https://www.fcc.gov/ narrowbanding-overview.

[3] [n.d.]. GNU Scientific Library. https://www.gnu.org/software/gsl/.

[4] [n.d.]. The LoRa Alliance Wide Area Networks for Internet of Things. https://www.lora-alliance.org/.

[5] [n.d.]. Multi-Use Radio Service (MURS). FCC. https: //www.fcc.gov/wireless/bureau-divisions/mobility-division/multiuse-radio-service-murs.

[6] [n.d.]. MURS Band Base Station Antenna. http://www.dbspectra.com/ products/140-150-mhz-6-dbd-omni-nf-antenna-ds1e06f36u-n/.

[7] [n.d.]. NLopt Documentation. https://nlopt.readthedocs.io/en/latest/.

[8] [n.d.]. NU Radio Manual and C++ API Reference. https://www. gnuradio.org/doc/doxygen/.

[9] [n.d.]. SIGFOXâĂŤThe Global Communications Service Provider for the Internet of Things (IoT). http://www.sigfox.com.

[10] [n.d.]. VHF MURS Band Data Radio / Telemetry Transceiver. https: //www.raveon.com/rv_m7_vm.html.

[11] [n.d.]. VHF Phantom Antenna. https://www.arcantenna.com/m2mantenna-hardware/category-phantom-antenna/vhf-low-profiledisguise-phantom-antenna/trat1500-m2m-140-300-mhz-white-lowprofile-omni-antenna.html.

[12] [n.d.]. VHF PHARAD Vest Antenna. http://www.pharad.com/vhfwearable-antenna.html.

[13] [n.d.]. White Space. https://www.fcc.gov/general/white-space.

[14] Omid Abari, Hariharan Rahul, Dina Katabi, and Mondira Pant. 2015. Airshare: Distributed coherent transmission made seamless. In Computer Communications (INFOCOM), 2015 IEEE Conference on. IEEE, 1742-1750.

[15] Shuchin Aeron and Venkatesh Saligrama. 2007. Wireless ad hoc networks: Strategies and scaling laws for the fixed SNR regime. IEEE Transactions on Information Theory 53, 6 (2007), 2044-2059.

[16] Richard Alimi, LI Li, Ramachandran Ramjee, Harish Viswanathan, and Yang Richard Yang. 2008. iPack: in-network packet mixing for high throughput wireless mesh networks. In IEEE INFOCOM 2008-The 27th Conference on Computer Communications. IEEE, 66-70.

[17] Ehsan Aryafar, Narendra Anand, Theodoros Salonidis, and Edward W Knightly. 2010. Design and experimental evaluation of multi-user beamforming in wireless LANs. In Proceedings of the sixteenth annual international conference on Mobile computing and networking. ACM, 197-208.

[18] Horia Vlad Balan, Ryan Rogalin, Antonios Michaloliakos, Konstantinos Psounis, and Giuseppe Caire. 2013. AirSync: Enabling distributed multiuser MIMO with full spatial multiplexing. IEEE/ACM Transactions on Networking (TON) 21, 6 (2013), 1681-1695.

[19] Cory Beard and William Stallings. 2015. Wireless Communication Networks and Systems. Pearson Education.

[20] MW Bercovici. 2006. FCC Narrowbanding Mandate: A Public Safety Guide for Compliance. 2006 (2006).

[21] Apurv Bhartia, Yi-Chao Chen, Lili Qiu, and George P Nychis. 2015. Embracing Distributed MIMO in Wireless Mesh Networks. In 2015 IEEE 23rd International Conference on Network Protocols (ICNP). IEEE, 66-77.

[22] Sourjya Bhaumik, Shoban Preeth Chandrabose, Manjunath Kashyap Jataprolu, Gautam Kumar, Anand Muralidhar, Paul Polakos, Vikram Srinivasan, and Thomas Woo. 2012. CloudIQ: A framework for processing base stations in a data center. In Proceedings of the 18th annual international conference on Mobile computing and networking. ACM, 125-136.
[23] SOLUTION BRIEF. [n.d.]. Intel Heterogeneous Network Solution Brief. ([n.d.]).

[24] Aleksandra Checko, Henrik L Christiansen, Ying Yan, Lara Scolari, Georgios Kardaras, Michael S Berger, and Lars Dittmann. 2015. Cloud RAN for mobile networksâĂŤA technology overview. IEEE Communications surveys \& tutorials 17, 1 (2015), 405-426.

[25] I Chih-Lin, Jinri Huang, Ran Duan, Chunfeng Cui, Jesse Xiaogen Jiang, and Lei Li. 2014. Recent progress on C-RAN centralization and cloudification. IEEE Access 2 (2014), 1030-1039.

[26] I Chih-Lin, Corbett Rowell, Shuangfeng Han, Zhikun Xu, Gang Li, and Zhengang Pan. 2014. Toward green and soft: A 5G perspective. IEEE Communications Magazine 52, 2 (2014), 66-73.

[27] Linglong Dai, Bichai Wang, Yifei Yuan, Shuangfeng Han, I Chih-Lin, and Zhaocheng Wang. 2015. Non-orthogonal multiple access for 5G: solutions, challenges, opportunities, and future research trends. IEEE Communications Magazine 53, 9 (2015), 74-81.

[28] Thomas R Dean, Mary Wootters, and Andrea J Goldsmith. 2018. Blind Joint MIMO Channel Estimation and Decoding. IEEE Transactions on Information Theory (2018).

[29] Aitor Del Coso, Umberto Spagnolini, and Christian Ibars. 2007. Cooperative distributed MIMO channels in wireless sensor networks. IEEE Journal on Selected Areas in Communications 25, 2 (2007), 402-414.

[30] Mischa Dohler, Athanasios Gkelias, and Hamid Aghvami. 2004. A resource allocation strategy for distributed MIMO multi-hop communication systems. IEEE Communications Letters 8, 2 (2004), 99-101.

[31] Adwait Dongare, Craig Hesling, Khushboo Bhatia, Artur Balanuta, Ricardo Lopes Pereira, Bob Iannucci, and Anthony Rowe. 2017. OpenChirp: A low-power wide-area networking architecture. In 2017 IEEE International Conference on Pervasive Computing and Communications Workshops (PerCom Workshops). IEEE, 569-574.

[32] Adwait Dongare, Revathy Narayanan, Akshay Gadre, Anh Luong, Artur Balanuta, Swarun Kumar, Bob Iannucci, and Anthony Rowe. 2018. Charm: exploiting geographical diversity through coherent combining in low-power wide-area networks. In 2018 17th ACM/IEEE International Conference on Information Processing in Sensor Networks (IPSN). IEEE, 60-71.

[33] Rashad Eletreby, Diana Zhang, Swarun Kumar, and Osman Yağan. 2017. Empowering low-power wide area networks in urban settings. In Proceedings of the Conference of the ACM Special Interest Group on Data Communication. ACM, 309-321.

[34] Tom Flanagan et al. 2011. Creating cloud base stations with TIâĂŹs KeyStone multicore architecture. Texas Instruments White Paper (2011).

[35] Adriana B Flores, Sadia Quadri, and Edward W Knightly. 2016. A scalable multi-user uplink for Wi-Fi. In 13th \{USENIX\} Symposium on Networked Systems Design and Implementation (\{NSDI\} 16). 179-191.

[36] Andrea Goldsmith. 2005. Wireless communications. Cambridge university press.

[37] Hao Guan, Troels Kolding, and Peter Merz. 2010. Discovery of cloudRAN. In Cloud-RAN Workshop, Vol. 2010.

[38] Mesud Hadzialic, Branko Dosenovic, Merim Dzaferagic, and Jasmin Musovic. 2013. Cloud-RAN: Innovative radio access network architecture. In Proceedings ELMAR-2013. IEEE, 115-120.

[39] Ezzeldin Hamed, Hariharan Rahul, Mohammed A Abdelghany, and Dina Katabi. 2016. Real-time distributed MIMO systems. In Proceedings of the 2016 ACM SIGCOMM Conference. ACM, 412-425.

[40] Ezzeldin Hamed, Hariharan Rahul, and Bahar Partov. 2018. Chorus: truly distributed distributed-MIMO. In Proceedings of the 2018 Conference of the ACM Special Interest Group on Data Communication. ACM, 461-475.

[41] Lars K Hansen and Guanghan Xu. 1997. A hyperplane-based algorithm for the digital co-channel communications problem. IEEE Transactions 
on information Theory 43, 5 (1997), 1536-1548.

[42] Harri Holma and Antti Toskala. 2012. LTE advanced: 3GPP solution for IMT-Advanced. John Wiley \& Sons.

[43] SM Riazul Islam, Nurilla Avazov, Octavia A Dobre, and Kyung-Sup Kwak. 2016. Power-domain non-orthogonal multiple access (NOMA) in $5 \mathrm{G}$ systems: Potentials and challenges. IEEE Communications Surveys \& Tutorials 19, 2 (2016), 721-742.

[44] Hu Jinling. 2010. TD-SCDMA/TD-LTE evolutionâĂŤGo Green. In 2010 IEEE International Conference on Communication Systems. IEEE, 301-305.

[45] Sachin Katti, Shyamnath Gollakota, and Dina Katabi. 2007. Embracing wireless interference: Analog network coding. In ACM SIGCOMM Computer Communication Review, Vol. 37. ACM, 397-408.

[46] Juho Lee, Younsun Kim, Hyojin Lee, Boon Loong Ng, David Mazzarese, Jianghua Liu, Weimin Xiao, and Yongxing Zhou. 2012. Coordinated multipoint transmission and reception in LTE-advanced systems. IEEE Communications Magazine 50, 11 (2012), 44-50.

[47] Yonghua Lin, Ling Shao, Zhenbo Zhu, Qing Wang, and Ravie K Sabhikhi. 2010. Wireless network cloud: Architecture and system requirements. IBM Journal of Research and Development 54, 1 (2010), $4-1$.

[48] Cheng Liu, Karthikeyan Sundaresan, Meilong Jiang, Sampath Rangarajan, and Gee-Kung Chang. 2013. The case for re-configurable backhaul in cloud-RAN based small cell networks. In 2013 Proceedings IEEE INFOCOM. IEEE, 1124-1132.

[49] Zhengxiang Ma, Michael Zierdt, John Pastalan, Arnold Siegel, Tod Sizer, Adriaan J de Lind van Wijngaarden, Praveen R Kasireddy, and Dragan M Samardzija. 2009. Radiostar: Providing wireless coverage over gigabit ethernet. Bell Labs Technical fournal 14, 1 (2009), 7-24.

[50] Mukundan Madhavan, Parul Gupta, and Malolan Chetlur. 2012. Quantifying multiplexing gains in a wireless network cloud. In 2012 IEEE International Conference on Communications (ICC). IEEE, 3212-3216.

[51] Konstantin Mikhaylov, Juha Petaejaejaervi, and Tuomo Haenninen. 2016. Analysis of capacity and scalability of the LoRa low power wide area network technology. In European Wireless 2016; 22th European Wireless Conference. VDE, 1-6.

[52] Shinobu Namba, Takashi Matsunaka, Takayuki Warabino, Shoji Kaneko, and Yoji Kishi. 2012. Colony-RAN architecture for future cellular network. In 2012 Future Network \& Mobile Summit (FutureNetw). IEEE, 1-8.

[53] John A Nelder and Roger Mead. 1965. A simplex method for function minimization. The computer journal 7, 4 (1965), 308-313.

[54] George Nychis, Thibaud Hottelier, Zhuocheng Yang, Srinivasan Seshan, and Peter Steenkiste. 2009. Enabling MAC Protocol Implementations on Software-Defined Radios.. In NSDI, Vol. 9. 91-105.

[55] Yao Peng, Longfei Shangguan, Yue Hu, Yujie Qian, Xianshang Lin, Xiaojiang Chen, Dingyi Fang, and Kyle Jamieson. 2018. PLoRa: a passive long-range data network from ambient LoRa transmissions. In Proceedings of the 2018 Conference of the ACM Special Interest Group on Data Communication. ACM, 147-160.

[56] Jonathan Perlstein, Thomas Dean, Mary Wootters, and Andrea Goldsmith. 2018. Fast Blind MIMO Decoding through Vertex Hopping. In 2018 52nd Asilomar Conference on Signals, Systems, and Computers. IEEE, 148-154.

[57] Tara Petrić, Mathieu Goessens, Loutfi Nuaymi, Laurent Toutain, and Alexander Pelov. 2016. Measurements, performance and analysis of LoRa FABIAN, a real-world implementation of LPWAN. In 2016 IEEE 27th Annual International Symposium on Personal, Indoor, and Mobile Radio Communications (PIMRC). IEEE, 1-7.

[58] Hariharan Shankar Rahul, Swarun Kumar, and Dina Katabi. 2012. JMB: scaling wireless capacity with user demands. In Proceedings of the ACM SIGCOMM 2012 conference on Applications, technologies, architectures, and protocols for computer communication. ACM, 235-246.

[59] Rapeepat Ratasuk, Benny Vejlgaard, Nitin Mangalvedhe, and Amitava Ghosh. 2016. NB-IoT system for M2M communication. In 2016 IEEE wireless communications and networking conference. IEEE, 1-5.

[60] Usman Raza, Parag Kulkarni, and Mahesh Sooriyabandara. 2017. Low power wide area networks: An overview. IEEE Communications Surveys \& Tutorials 19, 2 (2017), 855-873.

[61] Yuya Saito, Yoshihisa Kishiyama, Anass Benjebbour, Takehiro Nakamura, Anxin Li, and Kenichi Higuchi. 2013. Non-orthogonal multiple access (NOMA) for cellular future radio access. In 2013 IEEE 77th vehicular technology conference (VTC Spring). IEEE, 1-5.

[62] Jonathan Segel and Marcus Weldon. 2011. LightRadio portfolio: White paper 3. Alcatel-Lucent Bell Labs, Tech. Rep (2011).

[63] Souvik Sen, Naveen Santhapuri, Romit Roy Choudhury, and Srihari Nelakuditi. 2010. Successive interference cancellation: A back-ofthe-envelope perspective. In Proceedings of the 9th ACM SIGCOMM Workshop on Hot Topics in Networks. ACM, 17.

[64] Souvik Sen, Naveen Santhapuri, Romit Roy Choudhury, and Srihari Nelakuditi. 2013. Successive interference cancellation: Carving out MAC layer opportunities. IEEE Transactions on Mobile Computing 12, 2 (2013), 346-357.

[65] Osvaldo Simeone, Oren Somekh, H Vincent Poor, and Shlomo Shamai. 2008. Distributed MIMO in multi-cell wireless systems via finitecapacity links. In Communications, Control and Signal Processing, 2008. ISCCSP 2008. 3rd International Symposium on. IEEE, 203-206.

[66] Sanjib Sur, Ioannis Pefkianakis, Xinyu Zhang, and Kyu-Han Kim. 2016. Practical MU-MIMO user selection on 802.11 ac commodity networks. In Proceedings of the 22nd Annual International Conference on Mobile Computing and Networking. ACM, 122-134.

[67] Hina Tabassum, Md Shipon Ali, Ekram Hossain, Md Jahangir Hossain, and Dong In Kim. 2017. Uplink vs. downlink NOMA in cellular networks: Challenges and research directions. In 2017 IEEE 85th Vehicular Technology Conference (VTC Spring). IEEE, 1-7.

[68] Vamsi Talla, Mehrdad Hessar, Bryce Kellogg, Ali Najafi, Joshua R Smith, and Shyamnath Gollakota. 2017. Lora backscatter: Enabling the vision of ubiquitous connectivity. Proceedings of the ACM on Interactive, Mobile, Wearable and Ubiquitous Technologies 1, 3 (2017), 105.

[69] Shilpa Talwar, Mats Viberg, and Arogyaswami Paulraj. 1996. Blind separation of synchronous co-channel digital signals using an antenna array. I. Algorithms. IEEE Transactions on Signal Processing 44, 5 (1996), 1184-1197.

[70] Viktor Toldov, J Meijers, Román Igual-Pérez, Riaan Wolhuter, Nathalie Mitton, and Laurent Clavier. 2016. Performance evaluation of LoRa radio solution for PREDNET wildlife animal tracking project. In $L P$ WAN.

[71] Stavros Toumpis and Andrea J Goldsmith. 2003. Capacity regions for wireless ad hoc networks. IEEE Transactions on Wireless Communications 2, 4 (2003), 736-748.

[72] D Tse and P Vishwanathan. 2005. Multiuser capacity and opportunistic communication. Fundamentals of Wireless Communication (2005).

[73] Ambuj Varshney, Oliver Harms, Carlos Pérez-Penichet, Christian Rohner, Frederik Hermans, and Thiemo Voigt. 2017. Lorea: A backscatter architecture that achieves a long communication range. In Proceedings of the 15th ACM Conference on Embedded Network Sensor Systems. ACM, 18.

[74] Deepak Vasisht, Zerina Kapetanovic, Jongho Won, Xinxin Jin, Ranveer Chandra, Sudipta Sinha, Ashish Kapoor, Madhusudhan Sudarshan, and Sean Stratman. 2017. Farmbeats: An iot platform for data-driven agriculture. In 14th \{USENIX\} Symposium on Networked Systems Design and Implementation (\{NSDI\} 17). 515-529.

[75] Steven P Weber, Jeffrey G Andrews, Xiangying Yang, and Gustavo De Veciana. 2007. Transmission capacity of wireless ad hoc networks 
with successive interference cancellation. IEEE Transactions on Information Theory 53, 8 (2007), 2799-2814.

[76] Grace R Woo, Pouya Kheradpour, Dawei Shen, and Dina Katabi. 2007. Beyond the bits: cooperative packet recovery using physical layer information. In Proceedings of the 13th annual ACM international conference on Mobile computing and networking. ACM, 147-158.

[77] Dirk Wubben, Peter Rost, Jens Steven Bartelt, Massinissa Lalam, Valentin Savin, Matteo Gorgoglione, Armin Dekorsy, and Gerhard Fettweis. 2014. Benefits and impact of cloud computing on 5G signal processing: Flexible centralization through cloud-RAN. IEEE signal processing magazine 31, 6 (2014), 35-44.

[78] Kai Yang. 2012. Interference management in LTE wireless networks [Industry Perspectives]. IEEE Wireless Communications 19, 3 (2012), 8-9.

[79] Vivek Yenamandra and Kannan Srinivasan. 2014. Vidyut: exploiting power line infrastructure for enterprise wireless networks. In $A C M$ SIGCOMM Computer Communication Review, Vol. 44. ACM, 595-606. 